Revue d'histoire de l'Amérique française

ATS REVUE D.HISTOIRE DE L'AMÉRIQUE FRANÇAISE

\title{
Le contenu des registres paroissiaux canadiens du XVII ${ }^{\mathrm{e}}$ siècle
}

\section{Raymond Roy et Hubert Charbonneau}

Volume 30, numéro 1, juin 1976

URI : https://id.erudit.org/iderudit/303511ar

DOI : https://doi.org/10.7202/303511ar

Aller au sommaire du numéro

Éditeur(s)

Institut d'histoire de l'Amérique française

ISSN

0035-2357 (imprimé)

1492-1383 (numérique)

Découvrir la revue

Citer cette note

Roy, R. \& Charbonneau, H. (1976). Le contenu des registres paroissiaux canadiens du XVII ${ }^{\mathrm{e}}$ siècle. Revue d'histoire de l'Amérique française, 30(1), 85-97. https://doi.org/10.7202/303511ar d'utilisation que vous pouvez consulter en ligne.

https://apropos.erudit.org/fr/usagers/politique-dutilisation/ 


\title{
LE CONTENU DES REGISTRES PAROISSIAUX CANADIENS DU XVII ${ }^{\text {e SIÈCLE* }}$
}

\author{
RAYMOND ROY \\ Hubert Charbonneau \\ Département de démographie \\ Université de Montréal
}

Il n'y avait que 50 ou 60 immigrants français au Canada ${ }^{1}$ lorsque s'ouvrit le premier registre de Notre-Dame de Québec vers $1616^{2}$. Dès lors, l'accroissement des registres suivit celui de la population qui comptait 10000 personnes en 1680 et probablement 15000 en 1700. Le nombre de registres conservés jusqu'à ce jour passa, lui, de 5 en 1660 à 29 en 1680 puis à 46 à la fin du siècle ${ }^{3}$. Un peu plus de 200 prêtres y consignèrent 31000 actes dont 26000 concernent la population de souche européenne, les autres étant surtout des baptêmes d'Amérindiens. L'habitude de tenir registre remonte donc chez nous tout au début du peuplement et s'est maintenue jusqu'à nos jours, sans interruption.

Bien sûr, il y a eu des pertes: par disparition d'actes, de registres, mais aussi en raison du sous-enregistrement des décès.

* Ce texte n'aurait pu être préparé sans le généreux appui financier du Conseil des Arts du Canada (Programme Killam) et du Ministère de l'Education du Québec (Programme F.C.A.C.). Les auteurs remercient également de leur collaboration Pierre Beauchamp et André LaRose, assistants de recherche au Programme de recherche en démographie historique, de même que Louis Paquette, étudiant en informatique. Les calculs ont été faits à l'aide de l'ordinateur CYBER 74 du Centre de calcul de l'Université de Montréal.

1 Marcel Trudel, Histoire de la Nouvelle-France. Tome II': Le comptoir, (1604-1627) (Montréal et Paris, Fides, 1966) : 489.

2 André LaRose, Les registres paroissiaux au Québec aux XVII' et XVIII" siècles: introduction à l'étude d'une institution religieuse et civile. Mémoire de M.A. (histoire) (Université de Montréal, mars 1976), 109, note 1.

3 Ibid., 71; Hubert Charbonneau et Raymond Roy, "Généalogie, démographie et registres paroissiaux», Mémoires de la Société génécalogique canadiennefrançaise, XXVII, no 1 (janvier-mars 1976): 30.

RHAF, vol. 30, no 1 (juin 1976) 
Pour le XVII ${ }^{\mathrm{e}}$ seulement, nous avons estimé qu'environ 10000 actes manquent à l'appel, ce qui représente à peu près $28 \%$ des événements démographiques relatifs aux Canadiens de l'époque ${ }^{4}$. Ceci n'a cependant pas empêché la mesure sur échantillon des principaux paramètres démographiques des premières familles canadiennes et ne remet pas en cause non plus l'objectif final de notre projet de recherche, la reconstitution de la population entière, individu par individu. Trois recensements nominatifs et de riches sources d'appoint listes d'immigrants, archives notariales - nous permettront de corriger une bonne partie des lacunes. Il faut compter aussi sur la reconstitution des familles qui nous aidera à découvrir et à situer un grand nombre d'événements perdus. Celle-ci est presque achevée et nous nous engagerons bientôt dans l'élaboration et la critique des dossiers individuels ouverts à partir des fiches de famille.

Pour l'instant, nous avons voulu exposer le contenu des registres paroissiaux exploités jusqu'à maintenant. On comprendra mieux dès lors le succès des opérations de couplage automatique effectuées sur ces données ${ }^{5}$ et notre ambition d'en arriver prochainement à la reconstitution de la population canadienne du XVII ${ }^{\mathrm{e}}$ siècle. Nous soulignerons en particulier les variations qui affectent le contenu des actes dans le temps et dans l'espace.

La description qui suit porte sur un total de 26644 actes relatifs à la population de souche européenne seulement - dont 17624 baptêmes, 3689 mariages et 5331 sépultures provenant presque tous des registres conservés dans les presbytères. Quelques centaines seulement ont été relevés dans les archives civiles pour combler, au moins partiellement, certaines lacunes des séries paroissiales.

\section{Réglementation}

Au cours de la période qui nous intéresse ici, la tenue des registres a été marquée par une intervention législative qui ne tarda pas à faire sentir ses effets. Le 7 novembre 1678, le Conseil souverain de Québec enregistra l'Ordonnance promulguée à Saint-Germainen-Laye en 1667. Celle-ci obligeait les curés à tenir leurs registres en

4 Hubert Charbonneau, Vie et mort de nos ancêtres. Etude démographique (Montréal, Les Presses de l'Université de Montréal, 1975), 113.

5 Pierre Beauchamp, Raymond Roy et Jacques Légaré, «Reconstitution automatique des familles par le programme Hochelaga II», Population et Famille, 33 (1974-3): 1-40. 
double et à en remettre la copie à l'autorité civile une fois l'année expirée, sous peine d'amende. Elle rappelait aussi certaines règles déjà existantes dans la façon d'inscrire les divers types d'acte. On demandait spécifiquement aux rédacteurs de noter:

- dans les actes de baptême: la date de naissance, les noms .de l'enfant, du père et de la mère, du parrain et de la marraine.

- dans les actes de mariage: les nom, surnom, âge, qualité et demeure de chaque sujet d'acte, la présence de 4 témoins et la relation de parenté existante entre ceux-ci et les mariés.

- dans les actes de sépulture: la date de décès.

L'Ordonnance de 1667 introduisait aussi de nouveaux usages. Elle stipulait entre autres que dans les actes de mariage, les nouveaux époux devaient signer la minute, le curé étant tenu de mentionner dans l'acte s'ils avaient su le faire ou non. Un autre élément nouveau a trait aux décès. On exigeait que deux personnes signent l'acte de sépulture. C'était en même temps imposer la présence de deux témoins lors de cette cérémonie, ce qui n'avait jamais été explicite jusqu'alors ${ }^{6}$.

Il n'est pas possible de savoir dans quelle mesure les rédacteurs d'actes se sont conformés à l'obligation de tenir leurs registres en double. L'inventaire que nous avons entrepris dans les palais de justice ne révèle évidemment que ce qui subsiste et une part inconnue des lacunes que l'on y retrouve par rapport aux registres des paroisses tient à des pertes. Nous ne savons pas non plus si à partir de 1679, la fidélité des enregistrements s'est accrue ou, plus exactement, si le sous-enregistrement a diminué. La proportion d'événements démographiques perdus ne semble pas s'être modifiée tout au long du siècle ${ }^{7}$ mais là encore on ne peut départager les effets des deux causes qui composent cette mesure, à savoir les pertes accidentelles et le non-enregistrement d'événements. Il n'est cependant pas interdit de croire à un certain progrès de ce côté puisque le contenu des actes s'est nettement amélioré au lendemain de l'enregistrement de l'Ordonnance de Saint-Germain-en-Laye en 1678, comme nous le prouverons ci-dessous.

6 Pour plus de détails sur cette législation en particulier et sur l'ensemble de la réglementation relative à la tenue des registres paroissiaux, voir $R$. Le Mée, «La réglementation des registres paroissiaux en France». Société de démographie historique, Annales de démographie historique 1975: 433-472: Gérard Bouchard et André LaRose. "La réglementation du contenu des actes de baptêmes: mariage, sépulture au Québec, des origines à nos jours », RHAF, 30, no 1 (juin 1976) : 67-84.

7 Hubert Charbonneau, op. cit., 84 et 86 . 
Nous nous en tiendrons ici à l'examen de deux grandes périodes: avant et après le ler janvier 1680. L'analyse par décennie a montré que chacune de ces périodes était relativement homogène quant à la fréquence des informations que nous fournissent les registres paroissiaux sur les individus.

\section{Données relatives aux sujets d'acte}

La figure I rassemble l'information relative aux sujets d'acte des trois types. Examinons d'abord les baptisés dont les caractéristiques sont presque parfaitement relevées. Le prénom de l'enfant, le nom des parents et le jour du baptême sont pratiquement toujours mentionnés que ce soit avant ou après 1680. Il n'y a qu'un point faible, la date de naissance qui n'apparaît que dans $77 \%$ des cas. On note cependant un certain redressement de la situation à partir de 1680 , cette proportion passant de $72 \%$ à $80 \%$ de part et d'autre de l'Ordonnance.

Les caractéristiques des sujets d'acte de mariage sont en général moins bien déclarées mais l'essentiel y est. Les époux sont parfaitement identifiés et, plus de 9 fois sur 10 , on mentionne leurs parents ou le conjoint décédé. Leur état matrimonial est donc fort bien connu. Pour le reste, les lacunes sont nombreuses. L'âge au mariage n'apparaît qu'une fois sur quatre, la profession des hommes moins d'une fois sur cinq tandis que les fréquences relatives aux lieux de résidence et d'origine ainsi qu'aux signatures oscillent entre $40 \%$ et $60 \%$. Les changements sont cependant manifestes par suite de la promulgation de l'Ordonnance. Si l'on exclut l'origine, les modifications survenues de part et d'autre du moment de la législation vont toujours dans le sens d'un meilleur enregistrement et les progrès sont le plus souvent considérables: $38 \%$ pour l'âge, $9 \%$ pour les professions et $45 \%$ pour l'aptitude à signer. À côté de cela, les données concernant l'origine sont discordantes: contrairement à ce que l'on observe pour les autres éléments, l'origine des hommes est mentionnée beaucoup plus souvent que celle des femmes et la fréquence des informations diminue après 1679. L'explication de ce phénomène réside dans le fait que l'origine des personnes est déterminée par le lieu de résidence des parents. Or, d'une part, les Français sont deux fois plus nombreux que les Françaises parmi les sujets d'acte de mariage et, d'autre part, les immigrants forment une fraction de moins en moins importante dans l'ensemble des mariés au fur et à mesure que le siècle s'achève. En somme, la caractéristique «origine» nous 
FIGURE I

FRÉQUENCE DES DONNÉES RELATIVES AUX SUJETS D’ACTE DE LA PÉRIODE 1621-1699

SUJETS D'ACTE DE BAPTEMME

Nom et prenom

Mention des père et mere

Dates de baptême et de naissance

Date de baptếme seulement

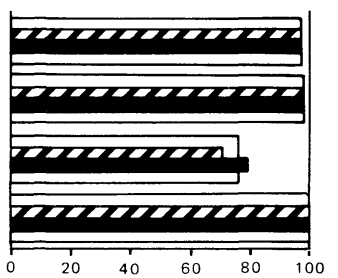

(Nombre relatif de sujets pour lesquels l'information existe)
LÉGENDE

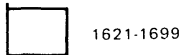

1621-1679

1680-1699

SUJETS D'ACTE DE MARIAGE

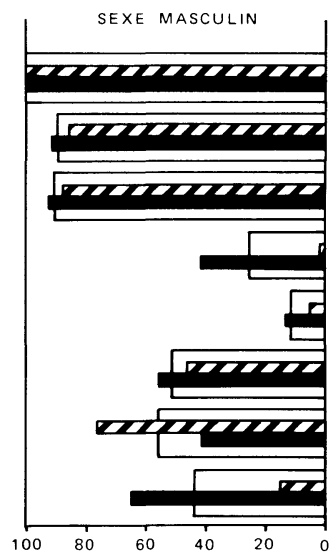

Nom et prénom Mention des père et mère ou du conjoint décédé

État matrimonial

Âge

Statut socio-professionnel

Résidence

Origine

Aptitude à signer

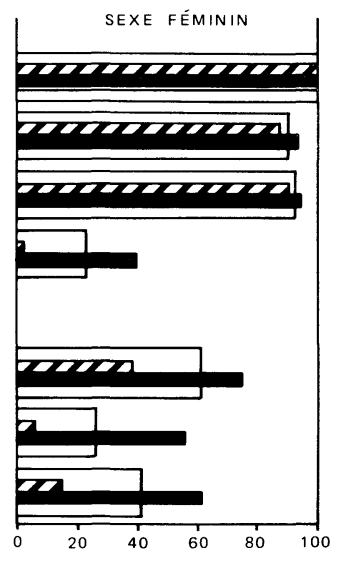

SUJETS D'ACTE DE SÉPULTURE

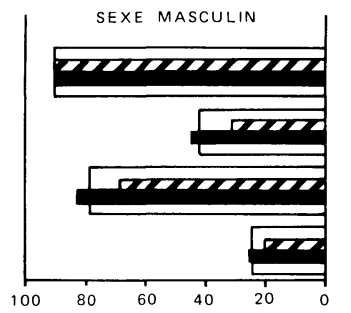

Nom et prénom

Mention des pere et mère

ou du conjoint du décédé

$$
\text { Âge }
$$

Résidence

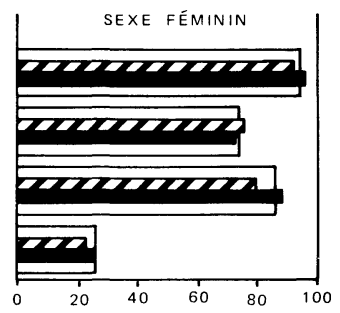


donne surtout une certaine approximation de la fréquence des sujets d'acte de mariage nés en France.

La situation des décédés est la moins satisfaisante: leurs caractéristiques nominatives apparaissent moins fréquemment que chez les autres sujets d'acte et les noms des parents et conjoints sont souvent oubliés. La résidence n'est mentionnée qu'une fois sur quatre, par contre l'âge est relevé dans $80 \%$ des cas. Ici encore, l'amélioration du contenu des actes après 1680 est sensible, notamment en ce qui a trait à l'identification des parents et à l'âge. On remarque aussi que les parents ou conjoints sont plus souvent présents lorsque le sujet d'acte est de sexe féminin. Ceci tient au fait qu'on ne déclare presque jamais les noms des parents ou conjoints des hommes décédés à l'âge adulte.

Malgré quelques déficiences, les données relatives aux sujets d'acte sont pour l'essentiel très satisfaisantes et peut-être exceptionnelles pour l'époque. L'identification des personnes est à toutes fins pratiques impeccable et les filiations, qui sont à la base des travaux de reconstitution des familles, ont été systématiquement relevées, sauf pour les décès. Les fréquences des autres caractéristiques des sujets d'acte sont plus faibles mais il ne faut pas exagérer la portée de ces lacunes qui sont parfois sans conséquence. Ainsi, l'enregistrement des lieux de résidence par exemple peut sembler superflu aux rédacteurs d'actes puisque, au départ, tous les actes sont situés dans le cadre d'une paroisse et qu'ils concernent surtout ses habitants. Signalons aussi une ambiguité inhérente à la collecte de cette information; l'expression «de cette paroisse» suit souvent une énumération de personnes et il est alors difficile voire impossible de déterminer celles auxquielles elle s'adresse en particulier: à une seule? à certaines d'entre elles? à l'ensemble des individus mentionnés? L'absence d'information étant préférable à une information douteuse ou fausse, le lieu de résidence n'a souvent été accolé qu'au dernier individu énuméré. Les résultats obtenus n'ont donc rien de surprenant.

Les défauts de déclaration d'âge au mariage et au décès sont plus gênants de sorte que nous ne connaîtrons jamais, ne serait-ce qu'approximativement, l'année de naissance d'un certain nombre d'immigrants n'apparaissant pas dans les recensements. Il en sera de même pour une fraction d'individus nés au Canada puisque $15 \%$ des enregistrements de naissance manquent. Pour ce qui est des professions et, plus largement, pour l'étude de la structure socio- 
professionnelle, il est évident que les registres paroissiaux ne constituent pas la source idéale du moins en ce qui regarde le $X V I I^{\mathrm{e}}$ siècle. Les «qualités» des personnes, quel que soit le rôle qu'elles jouent dans les actes - sujet, parents, témoin - sont rarement notées et celles qui le sont ont peu de chances d'être représentatives de l'ensemble.

\section{Données relatives aux parents et témoins}

Les caractéristiques des parents et témoins font l'objet de la figure II. Chez les premiers, l'information nominative est encore excellente bien que l'identification des conjoints décédés, dans les actes de mariage et de décès, soit un peu moins précise que celle des père et mère. Pour le reste, on observe que le lieu du domicile n'apparaît que dans $40 \%$ des cas et que, comme ailleurs, les professions ne sont qu'exceptionnellement déclarées. Quant aux changements intervenus par suite de l'Ordonnance, ils vont dans le sens d'un meilleur enregistrement, pour ce qui est des professions et des lieux de résidence, mais on note aussi une légère détérioration dans l'identification des conjoints des sujets d'acte. Il n'en demeure pas moins que le relevé des caractéristiques nominatives de ces personnes reste irréprochable dans 9 cas sur 10 après 1680 .

Outre les sujets d'acte et leurs parents, les registres paroissiaux du XVII ${ }^{\mathrm{e}}$ siècle identifient plus de 54000 témoins. À raison d'environ 2 par acte, ceux-ci représentent $30 \%$ des noms inscrits dans les registres. Signalons au passage que deux raisons ont motivé le relevé de ces mentions lors du dépouillement: premièrement, face à l'objectif d'en arriver à la reconstitution la plus complète possible de la population, ces mentions de témoins peuvent nous révéler l'existence d'individus qui n'apparaissent ni dans les actes - en tant que sujet, père, mère ou conjoint - ni dans les recensements. Ce risque n'est pas négligeable si l'on pense en particulier aux immigrants restés célibataires. Deuxièmement, les apparitions comme témoin nous aideront - c'est certainement sous ce rapport qu'elles seront le plus utile - à situer dans le temps des événements démographiques perdus, notamment des décès.

Lors des baptêmes, les témoins sont principalement les parrain et marraine. L'institution du parrainage remonte aux premiers siècles de l'Église, mais le premier texte connu prescrivant d'inscrire dans les actes les noms des personnes qui jouent ce rôle date du début du 
FIGURE II

FRÉOUENCE DES DONNÉES RELATIVES AUX PARENTS, CONUOINTS ET TÉNOINS MiENTIONNNÉS DANNS LES BAPTÊMES, MARIAGES ET SÉPULTURES DE LA PÉRIODE 1621-1699

\section{PARENTS ET CONJOINTS}
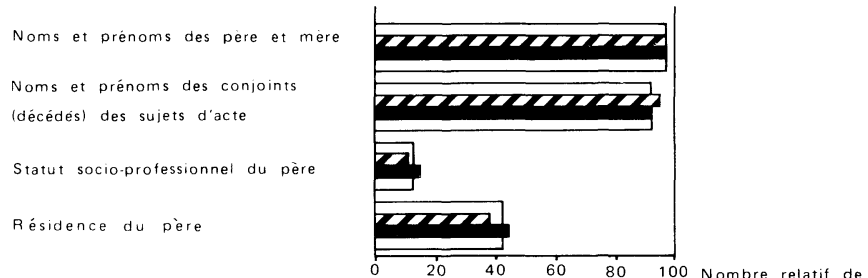

TÉMOINS

Nombre moyen par:

bap tême

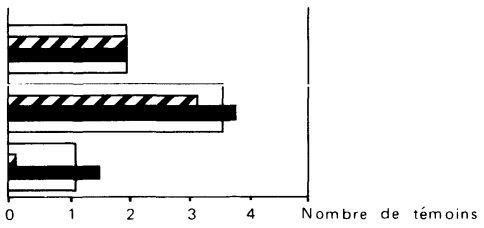

Nom et prénom

Statut socio-professionne

Résidence

LÉGENDE

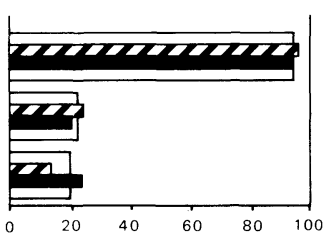

$\square \quad 1621.1699$

1621-1679

1680.1699

$X V^{\mathrm{e}}$ siècle $^{8}$. Cette règle a été bien suivie chez nous, comme en font foi les données de la figure II où l'on retrouve en moyenne 2 témoins par baptême. Il ne faut cependant pas croire que nous atteignions ici la perfection car on compte parmi ceux-ci un surplus d'hommes (18 295 contre 16939 ). On peut malgré cela estimer que près de 96\% des actes de baptême mentionnent les noms des parrain et mar-

'8 R. Le Mée, loc. cit.: 435. 
raine du sujet ${ }^{9}$. Remarquons par ailleurs qu'aucun changement ne s'est produit de ce côté au cours du siècle: la moyenne de témoins par baptême est la même avant et après 1680 .

Le nombre de quatre témoins devant assister au mariage a été fixé pour la première fois semble-t-il par l'Ordonnance de Blois en $1579^{10}$. Cette exigence a été reprise dans l'Ordonnance enregistrée à Québec un siècle plus tard. Apparemment, un rappel s'imposait et les rédacteurs d'actes en ont tenu compte. Si l'on en juge par les noms inscrits dans les actes, on trouve en moyenne 3,6 témoins par mariage. Ce nombre n'était que de 3,1 avant 1680 , mais à la fin du siècle, le minimum requis était presque atteint, la moyenne s'élevant à 3,8 .

En ce qui concerne les sépultures, la réglementation civile ou ecclésiastique ne renferme aucune allusion aux témoins avant 1667 et l'on ne s'étonnera pas de ne retrouver que de rarissimes témoins dans les actes d'avant 1680. Cependant, à la suite de l'intervention de 1678 qui imposait la présence de deux témoins, la situation change radicalement: les actes de sépulture enregistrés au cours des deux dernières décennies du siècle comptent en moyenne 1,5 témoins, sans compter la présence possible des parents qui lors de ce type d'événement peuvent servir de témoins. Ici, ils ne sont pas considérés à ce titre, autrement, la moyenne par acte pourrait très bien atteindre et même dépasser la norme. Signalons enfin que tant dans les mariages que dans les sépultures, les témoins sont presque toujours des hommes: ils forment respectivement $96 \%$ et $97 \%$ des mentions de témoins comprises dans ces actes.

Les fréquences des caractéristiques qui accompagnent les témoins sont au bas de la figure II. Leur identification est presque aussi complète que celle des parents et des sujets d'acte, noms et prénoms étant connus dans $95 \%$ des cas. Les professions et lieux de résidence n'apparaissent qu'une fois sur 5 à peu près. Conformément à ce qui a été observé précédemment, les données relatives à la résidence s'améliorent après 1680 mais curieusement, c'est la situation inverse qui se produit à propos des professions où nous croyons avoir affaire à un phénomène de sélection des témoins, phénomène qui se serait atténué avec le temps. Au début de la colonie,

\footnotetext{
y Si toutes les femmes témoins sont des marraines accompagnées d'un parrain, on a 16939 témoins de sexe féminin pour 17624 actes de baptême.

10 R. Le Mée, loc. cit. : 443.
} 
la petitesse des effectifs et aussi l'importance relative de l' «élite» rendaient plus facile la participation de celle-ci aux cérémonies. Or, on sait que les rédacteurs d'actes mettaient un soin particulier à noter les professions ou qualités de ces personnes. Plus tard, avec l'accroissement de la population, le recrutement des témoins a dû s'étendre à des couches plus larges où l'élite ne tenait plus la même place.

\section{Variations régionales}

Les ecclésiastiques qui ont assuré l'enregistrement des naissances, mariages et décès au XVII ${ }^{\mathrm{e}}$ siècle appartenaient à des communautés diverses (récollets, jésuites, sulpiciens, séculiers) que distinguaient des usages particuliers. On peut donc penser que ces usages affectaient la tenue des registres.

Nous n'avons pas encore regroupé les actes suivant l'appartenance des rédacteurs; néanmoins, la division du travail qui s'est opérée dans la colonie à l'époque permet non seulement d'évaluer les rendements de deux de ces groupes mais aussi de voir l'importance qu'ils donnaient aux divers éléments qui normalement devaient paraître dars les actes. Les deux groupes retenus sont les sulpiciens et les prêtres séculiers, qui ont rédigé respectivement $97 \%$ et $86 \%$ des actes de Montréal et de Québec "I. Il est donc permis d'attribuer les écarts éventuels entre les données de ces deux paroisses aux usages particuliers des prêtres qui les ont recueillies, même s'il faut s'abstenir de porter un jugement général sur les séculiers en particulier dont les comportements en cette matière devaient sûrement varier en fonction des réglementations propres à leur diocèse d'origine en France. La prudence s'impose pour une autre raison encore, la majorité des actes de Notre-Dame de Québec ayant été rédigés par un seul homme: Henri de Bernières. Cela dit, nous croyons que le rapprochement ne perd pas pour autant tout intérêt.

La figure III regroupe les éléments qui présentent les écarts les plus considérables entre les données des deux paroisses. On aurait pu imaginer que les différences se seraient manifestées toujours dans le même sens, en fonction du zèle plus ou moins grand de la part des communautés impliquées mais ce n'est pas le cas. Les écarts changent complètement de sens d'une caractéristique à l'autre: la situation de Québec est supérieure en ce qui concerne la mention des

11 Ces registres ont été tenus par les jésuites jusqu'en 1657 à Montréal et jusqu'en 1659 à Québec. 
FIGURE III

FRÉOUENCE DES DONNÉES DE LA PÉRIODE 1621-1699: QUÉBEC, MONTRÉAL, AUTRES PAROISSËS ET ENSEMBLE
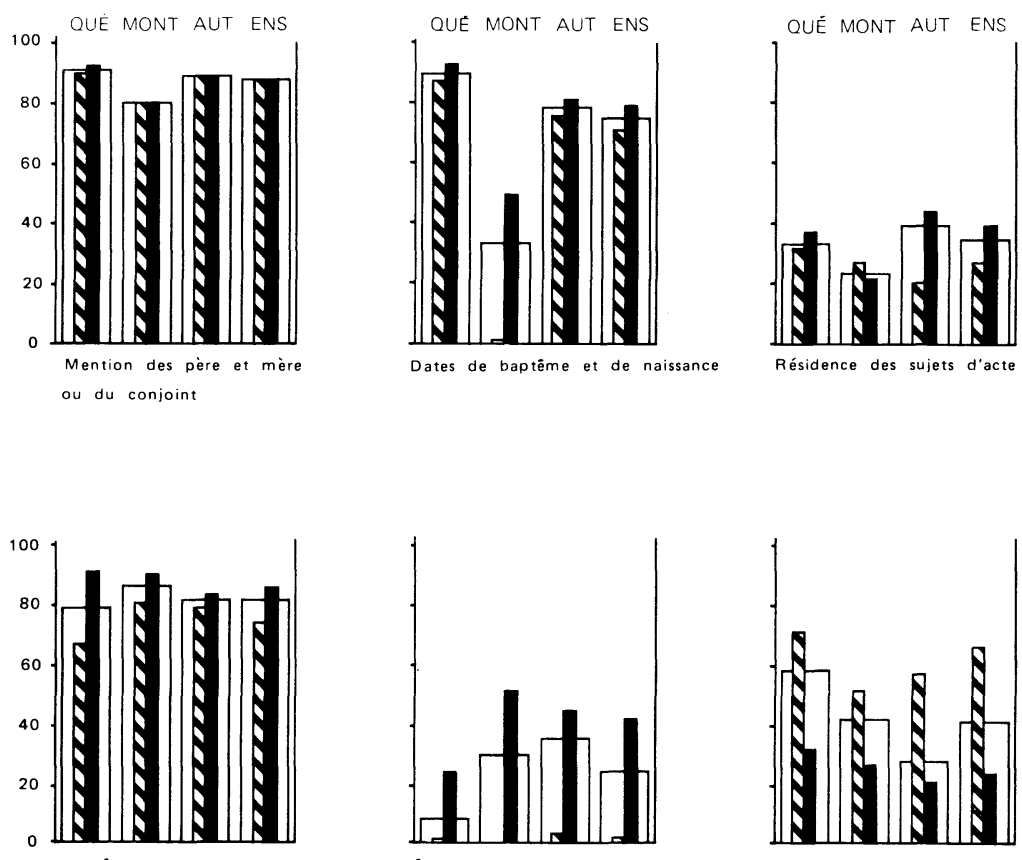

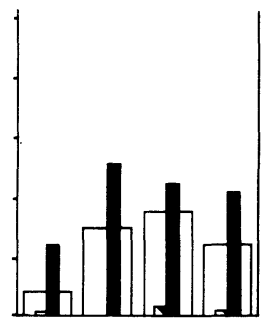

Age des sujets d'acte de mariage

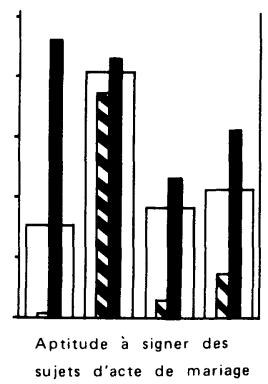

sujets d'acte de mariage

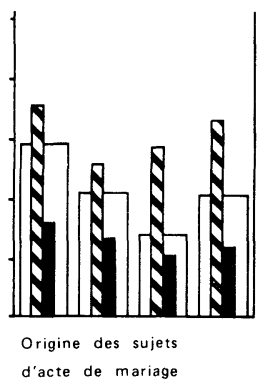

LÉGENDE

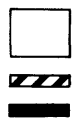

1621.1699

$1621 \cdot 1679$

1680.1699

Statut socio-profes.

sionnet des époux 
parents (ou du conjoint) ainsi que les dates de naissance et les lieux de résidence; par contre, pour les âges, les professions et l'aptitude à signer, le registre de Montréal est nettement meilleur.

Dans certains cas, les écarts s'expliquent par l'enregistrement précoce des informations. Il en est ainsi en ce qui regarde l'aptitude à signer des sujets d'acte de mariage, pour citer un cas extrême. À Montréal ce renseignement a été noté par les sulpiciens 3 fois sur 4 avant 1680, alors qu'à Québec, au cours de la même période, il n'apparaît à peu près jamais (à peine $1 \%$ des cas). Les différences dans les relevés des dates de naissance s'expliquent d'une façon analogue. Avant l'Ordonnance, elles sont mentionnées 9 fois sur 10 à Québec et une fois sur 100 à Montréal. Après 1680, les sulpiciens relèvent l'information avec plus de régularité soit une fois sur deux, alors qu'à Québec la situation s'améliore encore, la fraction des cas connus passant de $90 \%$ à $94 \%$.

Pour d'autres caractéristiques, les choses se passent autrement: en ce qui concerne l'âge ạı mariage, par exemple, les données antérieures à 1680 n'ont rien à voir avec la fluctuation observée entre les deux paroisses. L'Ordonnance de 1678 semble tout simplement avoir eu plus d'effet chez les sulpiciens. Outre les exemples mentionnés, la première explication (enregistrement précoce) vaut aussi pour les professions, l'âge des décédés, l'origine des sujets d'acte de mariage, alors que la seconde s'applique également aux données sur les lieux de résidence. Quant à la présence des parents des sujets d'acte, la supériorité de Québec sur Montréal est du même ordre de part et d'autre de la date de l'Ordonnance et, pour chaque paroisse, les fréquences des cas connus ne changent pas.

\section{CONCLUSION}

L'analyse des variables qui régissent ces phénomènes nécessiterait une étude approfondie des usages particuliers des communautés religieuses, car tout se passe comme si la réglementation officielle et générale en matière de tenue des registres paroissiaux était filtrée par des ensembles de coutumes propres aux groupes auxquels elle s'adresse. Ceci ne doit pas nier l'effet possible des variations dans les comportements individuels, mais il apparaît déjà que ce n'est pas là le facteur primordial.

Le fait que nos données soient sur bandes magnétiques nous permettra d'aller beaucoup plus loin dans cette direction et nous 
envisageons de faire éventuellement des regroupements d'actes non seulement par communauté mais aussi par rédacteur. Cet examen aidera grandement la critique des sources puisque celle-ci repose d'abord sur la connaissance des causes des lacunes. Malgré leur importance, ces dernières ne sont pas définitives, car nous ne manquons pas de ressources pour les combler. D'une part, il y a des sources complémentaires comme les recensements et les archives notariales; d'autre part, la science statistique nous permet de considérer comme pratiquement corrigées des lacunes bien repérées ${ }^{12}$. C'est pourquoi notre principal objectif, qui demeure la reconstitution de la population canadienne, ne sera guère affecté par les déficiences des registres paroissiaux.

12 Voir à ce sujet H. Charbonneau, op. cit., chap. 3. 